\title{
Prevention and Management of Gestational Diabetes Using Vitamin D Supplementation: An Overview and Appraisal of Clinical Trials
}

\author{
Aya Mousa \\ Monash Centre for Health Research and Implementation (MCHRI), School of Public Health \\ and Preventive Medicine, Monash University, Melbourne, VIC 3168, Australia; aya.mousa@monash.edu
}

Received: 20 October 2020; Accepted: 12 November 2020; Published: 17 November 2020

\begin{abstract}
A number of studies have examined the role of vitamin D in reproductive processes and disorders of pregnancy such as gestational diabetes mellitus (GDM). Although observational studies have linked maternal vitamin D deficiency with a plethora of adverse pregnancy outcomes including GDM, intervention trials generally do not support the use of vitamin D supplementation for GDM prevention or management. This narrative review provides an up-to-date overview and critical appraisal of randomised controlled trials (RCTs) to describe the current state of knowledge regarding the efficacy of vitamin D supplementation for preventing and/or managing GDM. Overall, although RCT data indicates a potential benefit of vitamin D in maternal glycaemic control, results are highly disparate and the data published to date have not conclusively established the efficacy of vitamin D in GDM prevention. There are, however, several limitations within the existing literature, including some considerable challenges that are unique to vitamin D trials, which should be carefully considered in the interpretation of the evidence and design of future studies. For now, many unanswered questions remain, and there is still a need for adequately powered and well-designed trials before routine supplementation can be recommended in the context of GDM.
\end{abstract}

Keywords: vitamin D; 25(OH)D; gestational diabetes mellitus; GDM; pregnancy; glycaemic control; randomized controlled trials; critical appraisal

\section{Introduction}

Vitamin D has a well-recognised role in calcium-phosphate homeostasis and bone mineralisation [1]. Accumulating evidence has implicated vitamin D in a number of disease states not related to bone health, including autoimmune [2,3], infectious [4,5], metabolic and cardiovascular diseases [6-9], as well as some cancers $[10,11]$ and neurological or mental health conditions [12-14], including schizophrenia [15]. Vitamin D has also been associated with reproductive processes and disorders of pregnancy [16,17]. Discovery of the nuclear vitamin D receptor (VDR) and metabolizing enzyme, $1 \alpha$-hydroxylase, in reproductive organs including the decidua, placenta, ovary, and endometrium, has seen an upsurge of studies examining potential links between vitamin D and adverse pregnancy outcomes [18]. In particular, there has been growing interest in the potential role of vitamin $\mathrm{D}$ in gestational diabetes mellitus (GDM), a common disorder of glucose intolerance with onset or first recognition occurring during pregnancy. However, the evidence is largely disparate and classifications for vitamin D deficiency or optimal concentrations during pregnancy remain contentious $[19,20]$.

Several lines of evidence support the potential involvement of vitamin D in the pathogenesis of GDM. In vitro studies have shown that active vitamin D has potent anti-inflammatory properties [21], and directly activates transcription of the insulin receptor gene [22], as well as regulating transcription of genes associated with placental invasion, normal implantation, and angiogenesis [23]. These studies 
posit that vitamin D deficiency may lead to increased inflammation and decreased insulin action, which could contribute to an increased risk of GDM. Observational studies have reported mixed results, with some finding inverse association setween maternal $25(\mathrm{OH}) \mathrm{D}$ concentrations and GDM risk factors including obesity [24], insulin resistance [25], dysglycaemia [26], dyslipidaemia [27], hypertension [28], and inflammation [29,30]; while others report no associations [31,32]. Similarly, some prospective studies link maternal vitamin D deficiency with an increased risk of incident GDM [33], while others do not [34-36].

Higher levels of evidence, such as that from randomised controlled trials (RCTs) have also produced mixed results, with some [37-40], but not all [41-43], supporting the use of vitamin D supplementation for preventing or managing GDM. These RCTs have been limited by small sample sizes and low statistical power, as well as low doses of vitamin D and variability in the baseline risk profiles of participants. Newly published trials [44-46] have sought to address these limitations, but the overall evidence from RCTs varies in scope, design, results and conclusions, making it difficult for decision-makers to reach definitive conclusions and recommendations for the use of vitamin $\mathrm{D}$ in clinical practice.

The aim of this paper is to provide an up-to-date overview of all published RCTs to describe the current state of knowledge regarding the efficacy of vitamin D supplementation for preventing and/or managing GDM. This review is not systematic and is not intended to introduce new data or conclusions, nor does it address all studies of vitamin $\mathrm{D}$ in pregnancy. Rather, the aim is to provide a comprehensive overview and critical appraisal of high-level evidence related to vitamin D and GDM and to present this in an objective, accessible and compact manner to aid in decision-making and future research endeavours.

\section{Methods}

A non-systematic search was conducted on electronic databases including PubMed, Google Scholar and Scopus using relevant search terms including: 'vitamin D'; 'supplements'; 'supplementation'; 'gestational diabetes'; 'GDM'; 'diabetes in pregnancy'; 'pregnancy'; 'reproductive'; 'randomized controlled trials'; 'RCTs', and combinations of these terms. Studies in English with an RCT design were collated for narrative synthesis if they reported on GDM incidence or glycaemic control during pregnancy (e.g., oral glucose tolerance test results or glycated haemoglobin A1c [HbA1c]) after vitamin D supplementation. Observational or non-randomized studies as well as studies reporting postpartum or other obstetric outcomes or not reporting at least one glycaemic parameter during pregnancy were excluded.

\section{Overview of Vitamin D}

\subsection{Vitamin D Physiology and Metabolism}

Vitamin D is a fat-soluble vitamin, which can be derived from dietary sources such as oily fish or fortified dairy products or from supplements in the form of ergocalciferol (vitamin $\mathrm{D}_{2}$ ) or cholecalciferol (vitamin $\mathrm{D}_{3}$ ). However, the primary source of vitamin $\mathrm{D}$ in humans is through skin exposure to ultraviolet-B (UVB) radiation [47]. The length of time needed to produce adequate vitamin D from skin exposure to sunlight depends on the strength of UVB rays (i.e., place of residence) and skin pigmentation [48].

Upon sun exposure, vitamin D is photobiosynthesised in the epidermis by 7-dehydrocholesterol, a normal byproduct of cholesterol synthesis [7]. Bound to a vitamin D binding protein (DBP), and to a lesser extent to albumin, this biologically inert form of vitamin $\mathrm{D}$ is transported to the liver where it undergoes hydroxylation to form 25-hydroxyvitamin $\mathrm{D}_{3}(25(\mathrm{OH}) \mathrm{D}$; the main measure of vitamin $\mathrm{D}$ status). A second hydroxylation occurs in the kidneys by the enzyme $1 \alpha$-hydroxylase (CYP27B1) to form 1,25-dihydroxyvitamin $\mathrm{D}_{3}\left(1,25(\mathrm{OH})_{2} \mathrm{D}_{3}\right)$, the biologically active form [49]. This active hormonal form, $1,25(\mathrm{OH})_{2} \mathrm{D}_{3}$, exerts pleiotropic biological functions by binding to a nuclear VDR, which is present 
in most human cells and tissues, including in the liver, kidney, ovary, pituitary, and endometrium, as well as in pancreatic $\beta$-cells [49]. The affinity of $1,25(\mathrm{OH})_{2} \mathrm{D}_{3}$ to bind to the VDR is 1000 times that of $25(\mathrm{OH}) \mathrm{D}$. However, the half-life of $25(\mathrm{OH}) \mathrm{D}$ is approximately 3 weeks, whereas $1,25(\mathrm{OH})_{2} \mathrm{D}_{3}$ has a shorter half-life of 4-6 h [49]. Vitamin D controls calcium absorption in the small intestine and works with parathyroid hormone (PTH) to mediate skeletal mineralization and maintain calcium homeostasis in the bloodstream [50].

\subsection{Measurement, Definition and Prevalence of Vitamin D Deficiency}

Measuring $1,25(\mathrm{OH})_{2} \mathrm{D}_{3}$ in serum is impractical due to a number of factors including its short half-life (hours), its highly lipophilic and unstable properties, and because it is present at picomolar concentrations within the circulation (a thousand-fold less than 25(OH)D) [51]. The formation and concentration of $1,25(\mathrm{OH})_{2} \mathrm{D}_{3}$ is also not directly regulated by vitamin $\mathrm{D}$ intake. Rather, it is regulated by other factors (such as serum PTH) and, even in the presence of severe vitamin D deficiency, $1,25(\mathrm{OH})_{2} \mathrm{D}_{3}$ levels may be normal or elevated due to an upregulation of the $1 \alpha$-hydroxylase enzyme. Serum $25(\mathrm{OH}) \mathrm{D}$ is thus currently considered the best indicator of vitamin D status [51].

Several commercially available methods are used for measuring vitamin D status, including competitive protein binding assays (CPBA), radioimmunoassays (RIA), enzyme-linked immunoassays (EIA or ELISA), random access automated assays using chemiluminescence technology (RAAA or chemiluminescent immunoassay [CLIA]), high-performance liquid chromatography (HPLC), and what is now considered to be the gold standard: liquid chromatography-tandem mass spectrometry (LC/MS) [7,51].

The blood concentration of $25(\mathrm{OH}) \mathrm{D}$ used to define deficiency remains an area of debate. The Institute of Medicine (IOM) [52] and National Institutes of Health (NIH) [53] suggest that circulating $25(\mathrm{OH}) \mathrm{D}$ concentrations $<50$ and $<25 \mathrm{nmol} / \mathrm{L}$ are considered deficient and severely deficient, respectively. These definitions would translate to $40-98 \%$ of pregnant women globally being deficient $(25(\mathrm{OH}) \mathrm{D}<50 \mathrm{nmol} / \mathrm{L})$, with $15-84 \%$ being severely deficient $(25(\mathrm{OH}) \mathrm{D}<25 \mathrm{nmol} / \mathrm{L})$ [54]. However, there is currently no consensus regarding optimal vitamin D levels during pregnancy, and the amount of supplementation required remains relatively unknown [19]. Epidemiological studies have postulated a U-shaped exposure-risk relationship between serum $25(\mathrm{OH}) \mathrm{D}$ concentrations and several disease outcomes (i.e., increased risk at both low $[<30 \mathrm{nmol} / \mathrm{L}]$ and high $[>75$ or $>125$ $\mathrm{nmol} / \mathrm{L}$ ] concentrations) [55]. These U-shaped curves may reflect biologically meaningful differences such as genetic variants, or may stem from confounding factors such as sun exposure or the use of vitamin D supplements by participants prior to study enrolment. Although both explanations are plausible, further investigations are needed to confirm these observations and how they may influence biological responses to vitamin $\mathrm{D}$.

Low vitamin D status is prevalent across all age-groups, geographic regions, and seasons [56]. Vitamin D concentration in blood varies cyclically over the course of the year in relation to genetic (gender, ethnicity, skin pigmentation, VDR polymorphisms) and environmental factors (sun exposure and sun safety practices, diet, food-fortification, use of vitamin D supplements). Data from the National Health and Nutrition Examination Surveys (NHANES) demonstrate a three-fold increase in the prevalence of severe vitamin D deficiency over the past 10-13 years in North America, with nearly $36 \%$ of the US population being deficient [57]. Similarly, an estimated $31 \%$ of adults in Australia have $25(\mathrm{OH}) \mathrm{D}$ concentrations $<50 \mathrm{nmol} / \mathrm{L}$, increasing to more than $50 \%$ during winter-spring and in people residing in Southern states [58].

The rising prevalence of vitamin D deficiency is due to several lifestyle changes including the global obesity epidemic and increase in sedentary indoor lifestyles, more common use of sunscreen and protective clothing due to fears of skin cancer, limited sources of vitamin D-rich foods or fortified food products, as well as insufficient amounts of vitamin D in dietary or prenatal supplements. Of concern is that populations with the greatest physiological needs for vitamin $\mathrm{D}$, such as pregnant women, neonates, and children tend to be at highest risk for vitamin D deficiency [59]. 


\subsection{Vitamin D in Pregnancy}

Discovery of the nuclear VDR and $1 \alpha$-hydroxylase enzyme in pregnancy-specific tissues such as decidua and placenta has generated substantial interest into the role of vitamin $\mathrm{D}$ in pregnancy. In normal pregnancy, maternal $1,25(\mathrm{OH})_{2} \mathrm{D}_{3}$ levels are twice as high by 12 weeks of gestation compared to the non-pregnant state, and peak at levels $2-3$ fold higher $\left(>700 \mathrm{pmol} / \mathrm{L}^{-1}\right)$ by the third trimester [60]. These concentrations would be toxic due to hypercalcaemia in a non-pregnant individual, yet, they appear to be essential in pregnancy with an apparent decoupling with calcium homeostasis. It is thought that the increase in $1,25(\mathrm{OH})_{2} \mathrm{D}_{3}$ may have an immunomodulatory rather than calcium-regulatory function (although it maintains the latter), and is highly dependent on bioavailability of the $25(\mathrm{OH}) \mathrm{D}$ substrate for conversion [60]. Thus, vitamin D deficiency in pregnancy is of particular importance because at no other time in life is $25(\mathrm{OH}) \mathrm{D}$ so closely linked with $1,25(\mathrm{OH})_{2} \mathrm{D}_{3}$ production.

Low vitamin $\mathrm{D}$ status in pregnancy predisposes to hypocalcaemia in the immediate postpartum period, and then to rickets in the following months. Infants breastfed by vitamin D-deficient mothers remain at high risk as the amount of vitamin $\mathrm{D}$ in breast milk correlates with maternal vitamin $\mathrm{D}$ status [61]. Conversely, vitamin D supplementation during pregnancy improves maternal vitamin D status and may positively affect the availability of vitamin D to the fetus and neonate [62]. This has important clinical implications and as such, the IOM recommends daily intakes of $600 \mathrm{IU}$ and $400 \mathrm{IU}$ of vitamin D for pregnant [52] and lactating women [61], respectively. However, these doses are likely inadequate to sufficiently raise blood $25(\mathrm{OH}) \mathrm{D}$ concentrations, particularly in the case of existing deficiency and limited sun exposure. In 2011, guidelines released by the Endocrine Society [63] suggested that all pregnant and lactating women with vitamin D deficiency receive 1000-2000 IU of vitamin D daily in addition to the $400 \mathrm{IU}$ provided in prenatal vitamins. These levels are consistent with current recommendations in Australia, where daily supplements containing 1000 or 2000 IU of vitamin $\mathrm{D}_{3}$ are recommended for pregnant women with $25(\mathrm{OH}) \mathrm{D}$ levels $<50$ and $<30 \mathrm{nmol} / \mathrm{L}$, respectively, while women with levels $>50 \mathrm{nmol} / \mathrm{L}$ should consume 400 IU daily as part of a pregnancy multivitamin [64].

In addition to bone health and infant transfer of vitamin D, maternal vitamin D deficiency has been associated with an increased risk of several other adverse pregnancy outcomes including GDM [59,65], pre-eclampsia [66], and preterm birth [67]. Although observational data supports a strong and consistent association between low vitamin D status and GDM, cause-effect relationships and the potential benefits of maternal vitamin $\mathrm{D}$ replenishment for the prevention and management of GDM remain unclear.

\section{Gestational Diabetes Mellitus}

Gestational diabetes mellitus (GDM) is a condition of glucose intolerance with onset or first recognition during pregnancy, and is characterised by insulin resistance and impaired insulin secretion [19]. In most women, GDM is asymptomatic and, depending on severity, it may be adequately controlled without medication (diet-controlled GDM), otherwise insulin and/or oral hypoglycaemic agents may be needed to achieve euglycaemia [68]. A diagnosis of GDM is generally made following an oral glucose tolerance test (OGTT) at 26-28 weeks gestation; however, the individuals targeted for screening as well as the timing and criteria used can vary. For instance, the American College of Obstetricians and Gynaecologists (ACOG) criteria requires two or more threshold values and use a $100 \mathrm{~g}$ glucose solution, while the Canadian Diabetes Association (CDA), the International Association of Diabetes and Pregnancy Study Groups Consensus Panel (IADPSG), and the World Health Organization (WHO) require only one threshold value and use a 75 g glucose solution [68-71]. Similarly, diagnostic cut-offs also differ, with a fasting glucose $\geq 5.3 \mathrm{mmol} / \mathrm{L}$ for CDA and ACOG and $5.1 \mathrm{mmol} / \mathrm{L}$ for IADPSG and WHO. A 1-h plasma glucose cut-off $\geq 10.0 \mathrm{mmol} / \mathrm{L}$ is adopted by all except CDA, which uses $10.6 \mathrm{mmol} / \mathrm{L}$; and a 2-h glucose cut-off $\geq 8.5 \mathrm{mmol} / \mathrm{L}$ is adopted by IADPSG and WHO, whereas the CDA cut-off is $\geq 9$ and ACOG is $\geq 8.6 \mathrm{mmol} / \mathrm{L}$. Only ACOG includes a 3-h plasma glucose, with a cut-off of $7.8 \mathrm{mmol} / \mathrm{L}$ [68-71]. 
An estimated $7 \%$ of pregnancies in the US were complicated by diabetes in 2009 and roughly $86 \%$ of these cases were women with GDM [72]. In Australia, GDM affects approximately $10-13 \%$ of pregnancies depending on the diagnostic criteria used [73]. GDM increases the risk of macrosomia, birth trauma, pre-eclampsia and neonatal hypoglycaemia [68]. The prevalence of GDM varies by ethnicity and is directly proportional to the prevalence of type 2 diabetes in the population [68]. Vitamin D has been implicated in both GDM and type 2 diabetes via its reported effects on insulin resistance and inflammation, and given the similar pathophysiology of these conditions [74,75]. However, the potential links between vitamin D status and GDM remain contentious, with several clinical trials attempting to clarify whether supplementation may reduce the risk of GDM or ameliorate its clinical presentation.

\section{Vitamin D and GDM: An Overview of Randomised Controlled Trials}

\subsection{Characteristics of Randomised Controlled Trials}

A total of 18 trials (20 articles with 18 unique samples) were identified and synthesized for the purpose of this review. The characteristics of these studies are summarised in Table 1. Most of the trials were conducted in Iran $(n=11)$, with two in China [39,76], and the remaining single studies conducted in India [41], Pakistan [77], Australia [42], and the United States (US) [43]. One study was a multi-centre RCT across seven European countries (United Kingdom, Ireland, Austria, Poland, Italy, Spain, and Belgium) [44]. Sample sizes ranged from 54 [37,38,78] to 500 [40] participants, but approximately half the studies ( $n=9$ RCTs) included $<100$ participants and nearly all $(n=16 / 18$ RCTs) included $\leq 200$ participants. Four trials had open-label designs [40,41,77,79], one was a single-blind RCT [44], while the rest were double-blind RCTs (Table 1).

In 13 of the 18 trials, the mean baseline $25(\mathrm{OH}) \mathrm{D}$ level of participants was $<50 \mathrm{nmol} / \mathrm{L}$, whereas three trials $[43,44,78]$ noted sufficient mean vitamin D levels at baseline $(25(\mathrm{OH}) \mathrm{D}<75 \mathrm{nmol} / \mathrm{L})$ and two $[76,80]$ did not report mean baseline vitamin D status. Twelve trials $[37,38,40-44,77,79-83]$ recruited women without GDM (healthy women or women at risk of GDM or preeclampsia) and aimed to examine whether vitamin D supplementation can prevent or reduce the risk of incident GDM or improve glycaemic measures. The remaining six trials $[39,45,46,76,78,84,85]$ included women diagnosed with GDM to examine the effects of vitamin D supplementation on glycaemic control or insulin resistance in these women (Table 1). 
Table 1. Characteristics of randomised controlled trials examining the efficacy of vitamin D supplementation on reducing incident GDM or improving maternal glycaemic control.

\begin{tabular}{|c|c|c|c|c|c|c|c|c|c|c|c|c|}
\hline No & $\begin{array}{l}\text { Author, Year, } \\
\text { Country }\end{array}$ & Study Design & Population & $\mathrm{n}$ & $\begin{array}{c}\text { Baseline } \\
\text { 25(OH)D } \\
(\mathrm{nmol} / \mathrm{L})\end{array}$ & $\begin{array}{l}\text { GDM } \\
\text { Criteria }\end{array}$ & Intervention & $\begin{array}{l}\text { Average } \\
\text { Daily } \\
\text { Dose (IU) }\end{array}$ & Control & $\begin{array}{l}\text { Micronutrient } \\
\text { Co-Interventions }\end{array}$ & Summary of Results & $\begin{array}{l}\text { 25(OH)D } \\
\text { Method }\end{array}$ \\
\hline 1 & $\begin{array}{l}\text { Asemi, } \\
\text { 2013a [37,38], } \\
\text { Iran }\end{array}$ & $\begin{array}{l}\text { Double-blind } \\
\text { parallel RCT }\end{array}$ & Healthy & 54 & 36.2 & NA & $\begin{array}{c}\text { Oral } \mathrm{VD}_{3} \\
400 \mathrm{IU} \text { daily } \\
\text { From } 25 \mathrm{GW} \text { for } 9 \text { weeks }\end{array}$ & 400 & Placebo & Folic acid, iron & $\begin{array}{l}\downarrow \text { FPG and insulin; } \\
\downarrow \text { HOMA-IR and } \\
\text { HOMA- } \beta \text {; } \\
\uparrow \text { QUICKI score; } \\
\text { GDM outcome NR }\end{array}$ & ELISA \\
\hline 2 & $\begin{array}{l}\text { Asemi, } \\
\text { 2013b [78], } \\
\text { Iran }\end{array}$ & $\begin{array}{l}\text { Double-blind } \\
\text { parallel RCT }\end{array}$ & GDM & 54 & 50.9 & ADA & $\begin{array}{c}\text { Oral } \mathrm{VD}_{3} \\
50,000 \text { IU twice } \\
\text { From 24-28 and } 27-31 \mathrm{GW}\end{array}$ & $\mathrm{NA}$ & Placebo & Folic acid, iron & $\begin{array}{c}\downarrow \text { FPG, insulin, } \\
\text { HOMA-IR and } \uparrow \\
\text { QUICKI; no effect on } \\
\text { HOMA- } \beta\end{array}$ & ELISA \\
\hline 3 & $\begin{array}{l}\text { Asemi, } \\
2015[84] \\
\text { Iran }\end{array}$ & $\begin{array}{l}\text { Double-blind } \\
\text { parallel RCT }\end{array}$ & GDM & 56 & 49.1 & ADA & $\begin{array}{c}\mathrm{Oral} \mathrm{VD}_{3} \\
50,000 \mathrm{IU} \mathrm{VD}_{3} \text { twice }+1000 \mathrm{mg} \\
\text { calcium/day } \\
\text { At mean } 25 \mathrm{GW} \text { and on day } 21 \\
\text { of intervention }\end{array}$ & $\mathrm{NA}$ & Placebo & Calcium & $\begin{array}{l}\downarrow \text { FPG, insulin, } \\
\text { HOMA-IR and } \uparrow \\
\text { QUICKI; no effect on } \\
\text { HOMA- } \beta\end{array}$ & ELISA \\
\hline 4 & $\begin{array}{l}\text { Corcoy, } 2020 \\
{[44], \text { Europe }} \\
(x 7 \text { countries })\end{array}$ & $\begin{array}{l}\text { Multi-centre } \\
\text { RCT }\end{array}$ & $\begin{array}{l}\text { Healthy with pre- } \\
\text { pregnancy BMI } \geq \\
\qquad 29 \mathrm{~kg} / \mathrm{m}^{2}\end{array}$ & 154 & 69.6 & IADPSG & $\begin{array}{c}{\mathrm{Oral} \mathrm{VD}_{3}}_{1600 \mathrm{IU} \text { daily }} \\
\text { From } \sim 15 \text { weeks until delivery }\end{array}$ & 1600 & Placebo & - & $\begin{array}{l}\text { Small } \downarrow \text { in FPG; no } \\
\text { effect on insulin, } \\
\text { HOMA-IR, } 1 \mathrm{~h} \text { or } 2 \mathrm{~h} \\
\text { glucose or insulin or } \\
\text { GDM risk }\end{array}$ & LC/MS \\
\hline 5 & $\begin{array}{l}\text { Hossain, } \\
2014[77] \\
\text { Pakistan }\end{array}$ & Open label RCT & Healthy & 200 & 13.7 & $\begin{array}{l}\text { O'Sullivan } \\
\text { and } \\
\text { Mahan }\end{array}$ & $\begin{array}{c}\text { Oral } \mathrm{VD}_{3} \\
4000 \mathrm{IU} \text { daily } \\
\text { From } 20 \mathrm{GW} \text { until delivery }\end{array}$ & 4000 & $\begin{array}{l}200 \mathrm{mg} \text { ferrous } \\
\text { sulfate }+600 \mathrm{mg} \\
\text { calcium daily }\end{array}$ & Iron, calcium & $\begin{array}{l}\text { No effect on GDM } \\
\text { incidence } \\
\text { (determined by } \\
\text { abnormal glucose } \\
\text { challenge test) }\end{array}$ & CLIA \\
\hline 6 & $\begin{array}{l}\text { Karamali, } \\
2015 \text { [81], } \\
\text { Iran }\end{array}$ & $\begin{array}{l}\text { Double-blind } \\
\text { parallel RCT }\end{array}$ & $\begin{array}{l}\text { Healthy women } \\
\text { at risk of } \\
\text { pre-eclampsia }\end{array}$ & 60 & 42.7 & NA & $\begin{array}{c}\mathrm{Oral} \mathrm{VD}_{3} \\
50,000 \mathrm{IU} \text { biweekly } \\
\text { From } 20 \mathrm{GW} \text { for } 12 \text { weeks }\end{array}$ & 3571 & $\begin{array}{l}\text { Placebo (+folic acid, } \\
\text { iron and MV) }\end{array}$ & $\begin{array}{l}\text { Folic acid, iron, } \\
\text { MV containing } \\
400 \mathrm{IU} \mathrm{VD} \\
3\end{array}$ & $\begin{array}{c}\downarrow \text { insulin, HOMA-IR } \\
\text { and HOMA- } \beta, \uparrow \\
\text { QUICKI; no effect on } \\
\text { FPG; GDM outcome } \\
\text { NR }\end{array}$ & ELISA \\
\hline
\end{tabular}


Table 1. Cont

\begin{tabular}{|c|c|c|c|c|c|c|c|c|c|c|c|c|}
\hline No & $\begin{array}{c}\text { Author, Year, } \\
\text { Country }\end{array}$ & Study Design & Population & $\mathrm{n}$ & $\begin{array}{c}\text { Baseline } \\
25(\mathrm{OH}) \mathrm{D} \\
(\mathrm{nmol} / \mathrm{L}) \\
*\end{array}$ & $\begin{array}{c}\text { GDM } \\
\text { Criteria }\end{array}$ & Intervention & $\begin{array}{c}\text { Average } \\
\text { Daily } \\
\text { Dose (IU) }\end{array}$ & Control & $\begin{array}{l}\text { Micronutrient } \\
\text { Co-Interventions }\end{array}$ & Summary of Results & $\begin{array}{l}\text { 25(OH)D } \\
\text { Method }\end{array}$ \\
\hline 7 & $\begin{array}{l}\text { Karamali, } \\
\text { 2018 [46] and } \\
\text { Jamilian, } 2019 \\
\text { [45], Iran }\end{array}$ & $\begin{array}{l}\text { Double-blind } \\
\text { parallel RCT }\end{array}$ & GDM & 60 & 33.8 & ADA & $\begin{array}{l}\text { Oral VD (type NR) } \\
200 \text { IU VD + } 400 \mathrm{mg} \text { calcium } \\
+4 \mathrm{mg} \text { zinc }+100 \mathrm{mg} \\
\text { magnesium daily (+usual } \\
\text { prenatal supplements) } \\
\text { From } 24-28 \text { GW for } 6 \text { weeks }\end{array}$ & 200 & $\begin{array}{c}\text { Placebo } \\
\text { (all women received } \\
\text { usual prenatal } \\
\text { supplements: } 1000 \\
\text { IU } \mathrm{VD}_{3}+400 \mathrm{ug} \\
\text { vitamin } \mathrm{B} 9+60 \mathrm{mg} \\
\text { iron daily) } \\
\end{array}$ & $\begin{array}{l}\text { Magnesium, } \\
\text { zinc, calcium, } \\
\text { B9, iron }\end{array}$ & $\begin{array}{l}\downarrow \text { FPG, insulin, } \\
\text { HOMA-IR } \\
\text { and } \uparrow \text { QUICKI }\end{array}$ & ELISA \\
\hline 8 & $\begin{array}{l}\text { Li, } 2016 \text { [39], } \\
\text { China }\end{array}$ & $\begin{array}{l}\text { Double-blind } \\
\text { parallel RCT }\end{array}$ & GDM & 103 & 40.5 & ADA & $\begin{array}{l}\text { Oral } \mathrm{VD}_{3} \text { (fortified yoghurt) } \\
\text { x2 servings daily of } 100 \mathrm{~g} \\
\text { yoghurt with } 500 \mathrm{IU} \mathrm{VD}_{3} \\
\text { From } 14 \mathrm{GW} \text { for } 16 \text { weeks } \\
\end{array}$ & 1000 & $\begin{array}{l}\text { Plain yoghurt (not } \\
\text { fortified) }\end{array}$ & - & $\begin{array}{l}\downarrow \text { FPG, insulin, } \\
\text { HOMA-IR, } \\
\text { and HOMA- } \beta\end{array}$ & NR \\
\hline 9 & $\begin{array}{l}\text { Mojibian, } \\
2015 \text { [40], } \\
\text { Iran }\end{array}$ & Open label RCT & Healthy & 500 & 38.2 & ADA & $\begin{array}{c}\mathrm{Oral} \mathrm{VD}_{3} \\
50,000 \mathrm{IU} \text { biweekly } \\
\text { From } 12-16 \mathrm{GW} \text { until delivery }\end{array}$ & 3571 & $400 \mathrm{IU} \mathrm{VD}_{3}$ daily & - & $\downarrow$ GDM risk & ELISA \\
\hline 10 & $\begin{array}{l}\text { Sablok, } 2015 \\
\text { [41], India }\end{array}$ & Open-label RCT & Heathy & 180 & 24 & NR & $\begin{array}{c}\text { Oral VD } \\
\text { BL VD >50 nmol/L: } 60,000 \mathrm{IU} \\
\text { once at } 20 \mathrm{GW} \\
\text { BL VD } 25-50 \mathrm{nmol} / \mathrm{L}: 120,000 \mathrm{IU} \\
\text { at } 20 \text { and } 24 \mathrm{GW} \\
<\text { BL VD }<25 \mathrm{nmol} / \mathrm{G}: 120,000 \mathrm{IU} \\
\text { at } 20,24,28 \text {, and } 32 \mathrm{GW}\end{array}$ & NA & No supplementation & - & $\begin{array}{l}\text { No effect on GDM } \\
\text { incidence }\end{array}$ & ELISA \\
\hline 11 & $\begin{array}{l}\text { Samimi, } \\
\text { 2016 [82], } \\
\text { Iran }\end{array}$ & $\begin{array}{l}\text { Double-blind } \\
\text { parallel RCT }\end{array}$ & $\begin{array}{l}\text { Healthy women } \\
\text { at risk of } \\
\text { pre-eclampsia }\end{array}$ & 60 & 40.5 & NR & $\begin{array}{c}{\text { Oral } \mathrm{VD}_{3}} \\
50,000 \mathrm{IU} \text { vitamin } \mathrm{D}_{3} \text { biweekly }+ \\
1000 \mathrm{mg} \mathrm{day}^{-1} \text { calcium } \\
\text { From } 20 \text { to } 32 \mathrm{GW}\end{array}$ & 3571 & Placebo & Calcium & $\begin{array}{l}\downarrow \text { FPG, insulin, } \\
\text { HOMA-IR, HOMA- } \beta \\
\text { and improved } \\
\text { QUICKI; GDM } \\
\text { outcome NR } \\
\end{array}$ & ELISA \\
\hline 12 & $\begin{array}{l}\text { Shahgheibi, } \\
\text { 2016 [83], } \\
\text { Iran }\end{array}$ & $\begin{array}{l}\text { Double-blind } \\
\text { parallel RCT }\end{array}$ & $\begin{array}{l}\text { Healthy women } \\
\text { at risk of GDM }\end{array}$ & 90 & 17.4 & $\begin{array}{l}\text { Specified } \\
\text { levels }\end{array}$ & $\begin{array}{c}\text { Oral VD (type NR) } \\
5000 \text { IU weekly } \\
\text { From } 12 \text { GW until } 26 \mathrm{GW}\end{array}$ & 714 & Placebo & - & $\begin{array}{l}\downarrow \text { GDM risk } \\
\text { (determined by } \downarrow \\
\text { proportion of } \\
\text { abnormal glucose } \\
\text { tolerance/challenge } \\
\text { tests at } 26 \mathrm{GW} \text { ) }\end{array}$ & $\begin{array}{l}\text { Liebermann } \\
\text {-Burchard } \\
\text { method }\end{array}$ \\
\hline
\end{tabular}


Table 1. Cont

\begin{tabular}{|c|c|c|c|c|c|c|c|c|c|c|c|c|}
\hline No & $\begin{array}{l}\text { Author, Year, } \\
\text { Country }\end{array}$ & Study Design & Population & $\mathrm{n}$ & $\begin{array}{c}\text { Baseline } \\
\text { 25(OH)D } \\
(\mathrm{nmol} / \mathrm{L}) \\
*\end{array}$ & $\begin{array}{c}\text { GDM } \\
\text { Criteria }\end{array}$ & Intervention & $\begin{array}{c}\text { Average } \\
\text { Daily } \\
\text { Dose (IU) }\end{array}$ & Control & $\begin{array}{l}\text { Micronutrient } \\
\text { Co-Interventions }\end{array}$ & Summary of Results & $\begin{array}{l}\text { 25(OH)D } \\
\text { Method }\end{array}$ \\
\hline 13 & $\begin{array}{l}\text { Soheilykhah, } \\
2013 \text { [79], } \\
\text { Iran }\end{array}$ & Open-label RCT & Healthy & 120 & 20.7 & NA & $\begin{array}{c}\text { Oral VD (type NR) } \\
\mathrm{I}_{1}=50,000 \text { IU monthly } \\
\mathrm{I}_{2}=50,000 \text { IU biweekly } \\
\text { From } 12 \mathrm{GW} \text { until delivery }\end{array}$ & $\begin{array}{l}\mathrm{I}_{1}=1667 \\
\mathrm{I}_{2}=3571\end{array}$ & 200 IU VD daily & - & $\begin{array}{c}\downarrow \text { HOMA-IR and } \\
\text { insulin in high-dose } \\
\text { (biweekly) group vs } \\
\text { controls; no effect on } \\
\text { FPG; GDM outcome } \\
\text { NR }\end{array}$ & CLIA \\
\hline 14 & $\begin{array}{l}\text { Tehrani, } \\
2017 \text { [80], } \\
\text { Iran }\end{array}$ & $\begin{array}{l}\text { Double-blind } \\
\text { parallel RCT }\end{array}$ & Healthy & 210 & NR & $\begin{array}{l}\text { Specified } \\
\text { levels }\end{array}$ & $\begin{array}{c}\mathrm{Oral} \mathrm{VD}_{3} \\
\text { 50,000 IU biweekly for } 10 \text { weeks } \\
\text { From 14-16 GW for } 10 \text { weeks }\end{array}$ & 3571 & $\begin{array}{c}\mathrm{C}_{1}=\text { Placebo } \\
\text { (omega-3 pearls); } \\
\mathrm{C}_{2}=\text { pregnant } \\
\text { women with VD > } \\
25 \mathrm{nmol} / \mathrm{L}\end{array}$ & - & $\begin{array}{l}\text { No effect on GDM } \\
\text { incidence }\end{array}$ & NR \\
\hline 15 & $\begin{array}{l}\text { Yap, } \\
\text { 2014 [42], } \\
\text { Australia }\end{array}$ & $\begin{array}{l}\text { Double-blind } \\
\text { parallel RCT }\end{array}$ & Healthy & 179 & 44.9 & ADIPS & $\begin{array}{c}\text { Oral } \mathrm{VD}_{3} \\
5000 \mathrm{IU} \text { daily } \\
\text { From }<20 \mathrm{GW} \text { until delivery }\end{array}$ & 5000 & $\begin{array}{l}400 \mathrm{IU} \text { Oral } \mathrm{VD}_{3} \\
\text { daily (low dose) }\end{array}$ & - & $\begin{array}{l}\text { No effect on GDM } \\
\text { incidence or glucose } \\
\text { levels }\end{array}$ & CLIA \\
\hline 16 & $\begin{array}{l}\text { Yazdchi, } \\
2016[85] \\
\text { Iran }\end{array}$ & $\begin{array}{l}\text { Double-blind } \\
\text { parallel RCT }\end{array}$ & GDM & 76 & 25.8 & IADPSG & $\begin{array}{c}\mathrm{Oral} \mathrm{VD}_{3} \\
50,000 \text { IU biweekly } \\
\text { From 24-28 GW for } 2 \text { months }\end{array}$ & 3571 & Placebo (paraffin oil) & Folic acid, iron & $\begin{array}{c}\qquad \text { FPG; no effect on } \\
\text { insulin or HOMA-IR; } \\
\uparrow \text { HbA1c in placebo } \\
\text { group }\end{array}$ & CLIA \\
\hline 17 & $\begin{array}{l}\text { Zerofsky, } 2014 \\
\text { [43], USA }\end{array}$ & $\begin{array}{l}\text { Double-blind } \\
\text { parallel RCT }\end{array}$ & Healthy & 57 & 69.6 & NR & $\begin{array}{c}\text { Oral } \mathrm{VD}_{3} \\
2000 \mathrm{IU} \text { daily } \\
\text { From } 20 \mathrm{GW} \text { until delivery }\end{array}$ & 2000 & $400 \mathrm{IU} \mathrm{VD}_{3}$ daily & - & $\begin{array}{l}\text { No effect on FPG or } \\
\text { measures of glucose } \\
\text { tolerance ( } 1 \mathrm{~h} \text { or } 2 \mathrm{~h} \\
\text { glucose) or HbA1c; } \\
\text { GDM outcome NR }\end{array}$ & LC/MS \\
\hline 18 & $\begin{array}{l}\text { Zhang, } \\
2016[76] \text {, } \\
\text { China }\end{array}$ & $\begin{array}{l}\text { Double-blind } \\
\text { multi-arm RCT }\end{array}$ & GDM & 133 & NR & NR & $\begin{array}{c}\text { Oral VD }_{3} \\
\mathrm{I}_{1}=200 \mathrm{IU} \text { daily } \\
\mathrm{I}_{2}=50,000 \mathrm{IU} \text { monthly } \\
\mathrm{I}_{3}=50,000 \mathrm{IU} \text { biweekly } \\
\text { From 24-28 weeks until delivery } \\
\left(\mathrm{I}_{1}\right) \text {, for } 25 \text { days }\left(\mathrm{I}_{2}\right) \text { or } 12.5 \text { days } \\
\left.\text { ( } \mathrm{I}_{3}\right)\end{array}$ & $\begin{array}{l}\mathrm{I}_{1}=200 \\
\mathrm{I}_{2}=1667 \\
\mathrm{I}_{3}=3571\end{array}$ & Placebo (sucrose) & - & $\begin{array}{l}\downarrow \text { insulin and } \\
\text { HOMA-IR with } \\
\text { high-and medium } \\
\text { dose but not with } \\
\text { low doses; ;o effect } \\
\text { on FPG }\end{array}$ & ELISA \\
\hline
\end{tabular}

* Reflects mean baseline 25(OH)D in the placebo/control group. ADA, American Diabetes Association; ADIPS, Australasian Diabetes in Pregnancy Society; BL, baseline; BMI, body mass index; C, control group; CLIA, chemiluminescent immunoassay; ELISA, enzyme linked immunosorbent assay; FPG, fasting plasma glucose; GDM, gestational diabetes mellitus; GW, gestational week/s; HbA1c, glycated haemoglobin A1c; HOMA-IR/ $\beta$; homeostatic model assessment of insulin resistance/ beta-cell function; I, Intervention group; IADPSG, International Association for Diabetes and Pregnancy Study Group; IU, international units; LC/MS, liquid chromatography/mass spectrometry; NR, not reported; NA, not applicable; QUICKI, qualitative RCT, randomised controlled trial; VD, vitamin $\mathrm{D}$. 
Dosage regimens varied substantially between studies, whereby seven trials used daily or weekly doses ranging between $200 \mathrm{IU}$ to $5000 \mathrm{IU}[37,38,42-46,77,83]$, and the remaining studies used biweekly or monthly doses of 50,000 IU [40,79-82,85], or bolus doses of 50,000 [37,78], 60,000 or 120,000 IU [41] at different frequencies and time points during the course of the pregnancy. Across the 15 studies using daily, biweekly or monthly doses, the average daily dose equates to approximately 2414 IU per day (Table 1). Only one study by Zhang et al. [76] compared daily doses with weekly and monthly bolus doses in a multi-arm trial, while Li et al. [39] used fortified yoghurt to administer vitamin D. All studies used oral cholecalciferol, with the exception of three studies which did not specify the type of vitamin $\mathrm{D}$ used $[45,46,79,83]$. Co-interventions were reported in approximately half the trials ( $\mathrm{n}=8 \mathrm{RCTs})$, most of which included calcium, iron and/or folic acid (Table 1), and it is likely that women across all trials were receiving multi-supplements as part of their routine antenatal care.

For the control groups, placebo was the comparator in 11 trials (Table 1), whereas four trials used low dose vitamin D (200 to 400 IU daily) [40,42,43,79] and the rest used either no supplementation [41], plain yoghurt [39], or calcium and iron [77] as the comparison/control group. Blood concentrations of 25(OH)D were assessed by ELISA in nine trials $[37,38,40,41,45,46,76,78,81,82,84]$, four used CLIA [42, $77,79,85]$ and only two employed the gold-standard LC/MS [43,44]. Of the remaining three studies, two did not report the method for determining circulating $25(\mathrm{OH}) \mathrm{D}[39,80]$ and one reported using the Liebermann-Burchard method [84].

\subsection{Efficacy of Vitamin D Supplementation for Reducing Incident GDM}

Regarding the effects of vitamin D supplementation on risk of incident GDM, results from the included RCTs were highly disparate. Of the 12 RCTs conducted in healthy women without GDM, only seven reported on incident GDM as an outcome [40-42,44,77,80,83]. Among these seven trials, five reported no change in the risk of GDM after supplementation with 1600 [44], 4000 [77], or 5000 IU [42] of cholecalciferol daily from $\leq 20$ weeks gestation or after receiving bolus doses of 50,000 IU biweekly from 14-16 weeks gestation [80] or 60,000 to 120,000 IU once, twice or four times from 20 weeks gestation [41]. Of note, two of these studies were open-label trials [41,77], one was single-blind [44] and all five were conducted in different geographical regions (Iran, India, Australia, Pakistan, Europe). All the trials also had different comparators including placebo, no supplements, iron and calcium, or low dose vitamin D. Hence, directly comparing these results or drawing conclusions as to the reasons for the lack of findings is challenging given the substantial heterogeneity between the study settings, designs and participants.

In the two remaining trials $[40,83]$, both of which were conducted in Iran, a reduced risk of GDM following vitamin D supplementation was reported. One of these trials was a double-blind RCT of 90 women which supplemented 5000 IU of vitamin D daily (type not reported) from 12 to 26 weeks gestation [83]. Here, Shahgheibi et al. [83] found a reduction in the proportion of abnormal glucose tolerance tests at 26 weeks gestation in the supplemented group compared with placebo. Participating women were at risk of GDM and given the small sample size, the generalisability of these results is questionable. The second trial was an open-label RCT and the largest trial to date as it included 500 women [40]. This trial found that women receiving biweekly supplementation with 50,000 IU of cholecalciferol from 12 weeks gestation had a significantly reduced risk of GDM, compared with a control group receiving the standard prenatal daily dose of $400 \mathrm{IU}$ [40]. These findings suggest that perhaps commencing supplementation at higher doses earlier in pregnancy may be more effective in preventing GDM. However, given the overall divergent results and null findings in most of the studies, there is a clear need for further well-designed trials using larger cohorts and a range of time points to clarify the benefits of vitamin D supplementation in reducing the risk of incident GDM, should any benefit exist. 


\subsection{Efficacy of Vitamin D Supplementation for Improving Glycaemic Control in Pregnant Women with and without GDM}

Twelve studies reported the effects of vitamin D supplementation on glycaemic parameters, of which six were in women with GDM $[39,45,46,76,78,84,85]$ and six were in healthy (or at risk) populations $[37,38,43,79,81,82]$.

The six studies in women with GDM were conducted in Iran $[45,46,78,84,85]$ and China $[39,76]$. All studies reported a reduction in fasting glucose, with the exception of one study by Zhang et al. [76] which reported no effect in 133 women receiving cholecalciferol at doses of 200 IU daily, 50,000 IU monthly or 50,000 IU biweekly compared with placebo. Similarly, all studies reported a reduction in insulin levels and insulin resistance measured by the homeostatic model assessment of insulin resistance (HOMA-IR), except for one study by Yazdchi et al. [85] which found no effect on these measures in 76 women receiving 50,000 IU biweekly from 24-28 weeks gestation for two months. However, Yazdchi et al. [85] did note a significant increase in HbA1c in the placebo group (compared with $\mathrm{HbA1}$ remaining stable in the vitamin $\mathrm{D}$ group), whereas $\mathrm{HbA1}$ c was not assessed in the remaining studies. Insulin sensitivity using the quantitative insulin sensitivity check index (QUICKI) was assessed in three studies from Iran, all of which found improvements after vitamin D supplementation, despite the different doses used (50,000 IU twice or 200 IU daily from $~ 24$ weeks gestation) and different regimens including vitamin D alone [78] or co-supplementing with calcium [84], or with magnesium, zinc and calcium [45,46]. Conversely, findings for HOMA of beta-cell function (HOMA- $\beta$ ) were inconsistent. One study from China [39] reported a reduction in HOMA- $\beta$ in 103 women receiving 1000 IU daily cholecalciferol in fortified yoghurt from 14 weeks gestation for 16 weeks, while two smaller studies $(n=54$ and $n=56)$ from Iran $[78,84]$ found no significant effect after two doses of $50,000 \mathrm{IU}$ cholecalciferol from 24 weeks gestation. Hence, in women with GDM, vitamin D appears to have some favourable effects on fasting glucose and insulin resistance, but results tend to vary for most outcomes, likely a reflection of the varied study designs, populations and supplementation regimens.

Of the six studies in healthy women, four were conducted in Iran, with one study in the US [43] and one across Europe [44]. The US study reported that $2000 \mathrm{IU}$ of cholecalciferol daily from 20 weeks gestation had no effect on insulin, HbA1c or fasting, 1-h or 2-h glucose post-OGTT [43]. Similarly, the multi-centre European trial [44] found a small reduction in fasting glucose but no effect on insulin, HOMA-IR, or 1-h or 2-h glucose post-OGTT after supplementation with 1600 IU of cholecalciferol daily from 15 weeks gestation until delivery. It should be noted that in both studies, participants were not vitamin D-deficient at baseline (mean $25(\mathrm{OH}) \mathrm{D}>50 \mathrm{nmol} / \mathrm{L}$ ). In contrast, all four studies from Iran $[37,38,79,81,82]$ reported that vitamin D supplementation reduced insulin levels and HOMA-IR. Fasting glucose was reduced in two studies $[37,38,82]$ while two reported no effect $[79,81]$; and three of the four studies $[37,38,81,82]$ showed a reduction in HOMA- $\beta$ and an increase in QUICKI (the fourth [79] did not measure these outcomes). Overall, the studies from Iran suggest an improvement in insulin resistance and glycaemic control with vitamin D supplementation, despite having quite different study regimens. For example, Asemi et al. [37,38] provided only $400 \mathrm{IU}$ of cholecalciferol daily from 25 weeks gestation, while the remaining three studies provided 50,000 IU biweekly from 12 [79] or 20 weeks gestation [81,82]. The doses in the latter studies average to approximately 3571 IU daily, a dose nine times higher than that provided in the studies by Asemi and colleagues [37,38]. Yet, with the exception of fasting glucose, these studies seem to be in agreement that vitamin D supplementation can improve measures of glycaemic control and insulin resistance in pregnant women without GDM, irrespective of the dose and duration of supplementation. This contrasts with the study by Soheilykhah et al. [79] which included three comparison groups (50,000 IU biweekly; 50,000 IU monthly, and $200 \mathrm{IU}$ daily [controls]), and reported that differences in glycaemic measures were only significant when comparing the high dose (biweekly) group with the controls, but not when comparing the lower dose (monthly) group with controls. Whether the effect of vitamin D on these outcomes is influenced by dosage regimens or other factors such as ethnicity, baseline risk profiles including 
glycaemic control or vitamin D status, or other study or population characteristics, remains unclear from the available evidence.

\section{Critical Appraisal and Considerations for Future Trials}

The literature summarised in this review represents the highest level of evidence of the efficacy of vitamin D supplementation for preventing or managing GDM. Despite a number of trials examining this topic over the last decade, results remain largely divergent and, in general, there is no strong evidence to support the use of vitamin D supplementation for GDM prevention or management. However, like observational studies that are limited by confounding and the inability to determine causation, RCTs are also subject to a number of limitations that likely contribute to these inconsistent results and threaten their external (e.g., low response rates) and internal (e.g., poor adherence, withdrawal, unblinding) validity. These limitations are summarised below and should be considered in the interpretation of results and in the design and execution of future trials.

First, RCTs often have low response rates and small sample sizes leading to poor external validity. This is demonstrated in the trials identified in this review, whereby half had less than 100 participants and most had less than 200 participants. The most recent trial by Corcoy et al. [44] indicated that 220 participants would be required to achieve statistical power to detect differences in insulin sensitivity and fasting glucose, with even more required to detect differences in a binary outcome such as GDM. Hence, most existing trials were underpowered which may explain some of the null results or inconsistencies identified in this review. Moreover, self-selection bias is problematic in RCT study designs since participants who volunteer to take part are more likely to be healthy or health conscious and less likely to be vitamin D-deficient [86]. While this may be less problematic in trials of pregnant women (since many women are typically more health conscious during pregnancy), there will always be some uncertainty as to whether results can be extrapolated to the general population. Together, these factors can reduce external validity and introduce bias, and are important to consider when interpreting the overall evidence.

A second key issue in vitamin D RCTs pertains to 25(OH)D levels and the common use of doses which are insufficient to adequately increase circulating $25(\mathrm{OH}) \mathrm{D}$ to target ranges. Indeed, a non-linear association between vitamin $\mathrm{D}$ status and disease risk has been demonstrated for all-cause mortality and cardiovascular disease, and may also exist for other conditions such as GDM [86]. This strongly suggests that vitamin $\mathrm{D}$ trials should include only participants with $25(\mathrm{OH}) \mathrm{D}$ concentrations below the inflection point where disease risk starts to rise, since supplementation would not be expected to reduce disease risk in people with $25(\mathrm{OH}) \mathrm{D}$ levels above this inflection point. This inflection point varies depending on the disease in question, with a previous review of the literature [87] concluding that the thresholds for beneficial vitamin D status could range from a 25(OH)D of $25 \mathrm{nmol} / \mathrm{L}$ for bone diseases to up to $100 \mathrm{nmol} / \mathrm{L}$ for cancer. Importantly, inflection points or thresholds for observing benefits in the context of pregnancy or GDM have not been established. Nevertheless, the presence of vitamin D-sufficient individuals in a trial is sure to weaken its statistical power because their risk of disease remains unchanged irrespective of whether they receive vitamin D or placebo [86]. This, in turn, can mask the potential benefits of vitamin $\mathrm{D}$, leading to erroneous results and conclusions. The trials reviewed herein demonstrate this concept to some extent, since the two trials $[43,44]$ which found no effect on most or any of the measured glycaemic outcomes (including GDM risk) both reported a non-deficient mean baseline 25(OH)D level $(69 \mathrm{nmol} / \mathrm{L})$. Much like we would not include slim individuals in a trial examining obesity reduction, if we are to optimize the detection of potential benefits from vitamin D supplements, participants with sufficient vitamin D levels should not be included in trials examining the efficacy of vitamin D supplementation. This is even more pertinent in vitamin D trials as baseline $25(\mathrm{OH}) \mathrm{D}$ can influence the half-life of ingested vitamin $\mathrm{D}$, since higher concentrations can lead to faster catabolism of vitamin D to maintain equilibrium [88]. Therefore, despite a lack of consensus regarding the inflection points or optimal concentrations required to achieve health gains 
in pregnancy, at the very least, RCTs should aim to recruit participants with vitamin D deficiency $(<50 \mathrm{nmol} / \mathrm{L})$ and provide supplementation regimens adequate for achieving sufficiency $(>75 \mathrm{nmol} / \mathrm{L})$.

A detailed discussion of this issue is provided by Heaney [89], where, in addition to recruiting participants who are vitamin D-deficient, it is recommended that trials provide a sufficiently large dose of vitamin $\mathrm{D}$ to shift their status into the range where disease risk decreases (or benefit increases), and assess temporal changes in vitamin D status to verify the adequacy of the dose and adherence. As noted above, the exact 'point' at which disease risk shifts can vary depending on the disease in question and is generally not known, especially in pregnancy. Moreover, the exact dose and regimen required to achieve sufficiency also varies and can heavily influence results. For instance, bolus doses may lead to short-lived peaks in intracellular vitamin $\mathrm{D}$, followed by longer periods of insufficient vitamin $\mathrm{D}$ levels, resulting in an overall absence of physiological effects [90]. Similarly, a daily dose of 400 IU for several months will achieve little or no detectable effect on circulating vitamin D, whereas extended periods of 2000-6000 IU daily can maintain stable concentrations in the range of $25-100 \mathrm{nmol} / \mathrm{L}$ in a linear fashion [91,92]. These factors have clear implications in vitamin $\mathrm{D}$ trials but remain to be fully understood within the pregnancy context, emphasising the need for further research to clarify the optimal 25(OH)D concentrations for pregnancy-related benefits and the doses needed to achieve these.

An important caveat to note with regard to $25(\mathrm{OH}) \mathrm{D}$ levels is the ethical dilemma introduced by providing placebo in the presence of deficiency. Ethics review committees may require that participants in the control arm also be given vitamin D, particularly in the case of pregnancy where deficiency (especially severe deficiency) can pose undue risk to the mother or fetus. While the vitamin D doses in prenatal vitamins are usually low (e.g., 200-400 IU daily), they are still high enough to shift control participants out of severe vitamin D deficiency, hence impacting the power to detect differences as noted above. An alternative would be to store blood samples at baseline for measurement of 25(OH)D later once the trial is completed. However, while this can be justified on the basis of equipoise about the effect of vitamin D supplementation on most outcomes, this is not possible during pregnancy as treatment of severe deficiency is critical for preventing infantile complications such as rickets. The use of multivitamins or low dose vitamin D supplements in the control group is therefore inevitable and will always be an issue for vitamin D trials in pregnancy, one which requires appropriate planning and consideration in the design phase of these trials.

Closely linked to the point above regarding baseline 25(OH)D status and target levels, the third issue with vitamin $\mathrm{D}$ trials, both in and outside of pregnancy, is that they do not follow the traditional model for conducting clinical trials, where the evaluation of interventions is entirely under the control of clinicians and researchers. Unlike drug trials for example, participants in vitamin D trials have easy access to vitamin D supplements, widely available from pharmacies, supermarkets and online. This contributes to a high risk of contamination among control participants, should they take supplements outside the trial protocol [86]. Contamination can in turn influence 25(OH)D levels and, as described above, alterations in $25(\mathrm{OH}) \mathrm{D}$ levels can lead to a number of problems with analysing and interpreting RCT results. It can be argued that self-purchased vitamin D is likely to be similar in both intervention and control arms of a double-blind trial, thereby cancelling out any differences in effects between groups. In reality however, this is not the case since taking supplements will reduce the proportion of vitamin D-deficient participants in the control arm (without really impacting the intervention arm) and hence reduce the chances of detecting a beneficial effect, particularly if the association between vitamin D status and disease risk is non-linear as has been demonstrated [86]. Participants can also have their vitamin D levels tested relatively easily, which is especially problematic among pregnant women who often have their vitamin D levels checked as part of routine antenatal care. This can risk unblinding and further exacerbate contamination should they decide to take supplements or make lifestyle changes such as increasing their sun exposure or intake of vitamin D-fortified foods to self-treat their deficiency. These limitations can reduce the internal validity of RCTs and influence their findings, and may in part explain some of the discrepancies demonstrated in the literature. To counter these issues, trials need to incorporate close monitoring of $25(\mathrm{OH}) \mathrm{D}$ status, with frequent sampling and 
testing over the course of a trial to assess potential protocol violations, but this comes with substantial costs and time commitments. Of note, none of the included trials measured vitamin D status at different time points across the course of pregnancy, hence the above problems could have been encountered and remained undetected.

Fourth, the clinical trials reviewed herein demonstrate substantial heterogeneity and variations in methodological rigour that should be considered in the interpretation of their findings. For instance, there are considerable differences in study designs, populations and settings, including varying cut-off points used to define vitamin $\mathrm{D}$ sufficiency and/or deficiency, different diagnostic criteria for GDM (ADA, IADPSG, etc.), different assays used to determine 25(OH)D levels (with only two studies using the gold-standard LC/MS), and different geographies and population groups studied, with the majority of trials $(n=11 / 18)$ being conducted in Iran, limiting generalisability (Table 1$)$. Participants also had different risk profiles at baseline, with some having GDM or being classified as high-risk and others being relatively healthy, hence, direct comparisons become challenging. Some studies $[78,84]$ noted significant differences in baseline glycaemic parameters between intervention and control groups (e.g., the placebo group was less insulin-resistant at baseline), making their results difficult to interpret. It is also important to consider the selection of factors to be controlled in an experimental setting, since some aspects such as body mass index, ethnicity, and increased maternal age can increase the risk of both GDM and vitamin D deficiency [93]. Other factors strictly related to vitamin D, such as endogenous biosynthesis (sun exposure) and amounts of dietary intake, should be taken into account when interpreting results from vitamin D trials. Yet, lifestyle factors such as these have seldom been incorporated in the existing trials and may explain some of the variations observed in the evidence.

Finally, there remain uncertainties regarding the functions of vitamin $\mathrm{D}$ at different stages of pregnancy, how these functions may be related to different clinical outcomes, and the appropriate 'critical windows' for measuring and treating deficiency or insufficiency in line with relevant pregnancy outcomes. For instance, gestational age is a crucial consideration for intervention studies since repletion needs to be restored prior to observing changes in the outcome of interest. Although blood 25(OH)D levels may promptly respond to supplementation (assuming sufficient doses), peripheral cellular deficiency may require more time. Intervention studies designed during late pregnancy may therefore not have sufficient time to restore the physiological conditions required for the prevention of dysfunction, or indeed, GDM [94]. The short durations of many of the existing trials (i.e., weeks or months) may also be ineffective in altering the pathogenic pathways of disorders such as GDM, which is thought to have initial manifestations from the very early stages of pregnancy [94]. This may explain some of the null or inconsistent findings from existing studies since, of the 18 included trials, only three $[40,79,83]$ started supplementation in the first trimester (i.e., from $\sim 12$ weeks), while the majority $(n=12 / 18$ RCTs) commenced at or after 20 weeks gestation, with many lasting less than three months.

When considering the above limitations, coupled with small sample sizes, lack of statistical power, open-label designs, and the use of low dose vitamin D supplements in the control groups (which is largely unavoidable in pregnancy), the equivocal findings presented in this review are not surprising. This is further compounded by a lack of defined knowledge around the appropriate doses or optimal concentrations of vitamin $\mathrm{D}$, and the duration of supplementation necessary to appreciate any benefits, particularly during pregnancy when the vitamin D system undergoes substantial adaptations to support increased maternal and fetal requirements. Co-supplementation was also often used, making it impossible to isolate the effects of vitamin $\mathrm{D}$ alone. This is particularly important when considering co-supplementation with magnesium since magnesium is an integral cofactor in the enzymatic conversion of vitamin $\mathrm{D}$ to $25(\mathrm{OH}) \mathrm{D}$ and $1,25(\mathrm{OH}) \mathrm{D}$, and is present in most pregnancy multivitamins [95]. Studies also varied in their supplementation regimens, ranging between daily, weekly, biweekly, monthly or intermittent supplementation of varying doses. As mentioned earlier, these regimens may influence the measured outcomes since bolus doses can be absorbed rapidly and become undetectable, while a steady state can be achieved by supplying constant doses of vitamin $\mathrm{D}$ for 
3-4 months [96]. In addition, as outlined in this review, there are many uncertainties regarding whether vitamin $\mathrm{D}$ is only effective in certain subgroups or whether a certain threshold exists at which vitamin $\mathrm{D}$ ceases to incur additional benefits. It is possible that vitamin $\mathrm{D}$ may have differential effects in women of different ethnicities, age groups or baseline risk profiles, but this is yet to be fully delineated. Finally, there is a need to ascertain the long-term effects of vitamin D supplementation during pregnancy to determine whether improvements in glycaemic control following supplementation may translate into a reduced risk of type 2 diabetes or recurrent GDM in subsequent pregnancies. Taken together, while the existing research indicates potential benefits of vitamin $\mathrm{D}$ for glycaemic control in pregnancy but not for GDM prevention per se, more conclusive evidence is needed to confirm these findings and to determine the mechanistic pathways underlying the protective features of vitamin $\mathrm{D}$, if any.

\section{Conclusions}

In summary, RCT data published to date have not conclusively established the efficacy of vitamin $\mathrm{D}$ in the prevention or management of GDM. Importantly, there are several challenges and limitations within the existing literature that should be carefully considered in the interpretation of the evidence. In light of these limitations, there is currently insufficient evidence to support the use of vitamin $\mathrm{D}$ for preventing or managing GDM, despite some clinical trials indicating that vitamin D may improve glycaemic parameters in pregnancy. For now, there remain unanswered questions regarding the potential use of vitamin D supplementation as a GDM prevention or management strategy, and there is a need for large-scale, adequately powered, and well-designed RCTs addressing the above limitations before routine supplementation can be recommended for this purpose.

Author Contributions: Conceptualization, data curation, writing—original draft preparation, review and editing, A.M. The author has read and agreed to the published version of the manuscript.

Funding: This research received no specific or external funding.

Acknowledgments: A.M. is supported by a Peter Doherty Biomedical Research Fellowship provided by the National Health and Medical Research Council (NHMRC) of Australia.

Conflicts of Interest: The authors declare no conflict of interest.

\section{References}

1. Charoenngam, N.; Shirvani, A.; Holick, M.F. Vitamin D for skeletal and non-skeletal health: What we should know. J. Clin. Orthop. Trauma 2019, 10, 1082-1093. [CrossRef] [PubMed]

2. Hyppönen, E. Vitamin D and increasing incidence of type 1 diabetes-Evidence for an association? Diabetes Obes. Metab. 2010, 12, 737-743. [CrossRef] [PubMed]

3. Souberbielle, J.-C.; Body, J.-J.; Lappe, J.M.; Plebani, M.; Shoenfeld, Y.; Wang, T.J.; Bischoff-Ferrari, H.A.; Cavalier, E.; Ebeling, P.R.; Fardellone, P. Vitamin D and musculoskeletal health, cardiovascular disease, autoimmunity and cancer: Recommendations for clinical practice. Autoimmun. Rev. 2010, 9, 709-715. [CrossRef] [PubMed]

4. Urashima, M.; Segawa, T.; Okazaki, M.; Kurihara, M.; Wada, Y.; Ida, H. Randomized trial of vitamin D supplementation to prevent seasonal influenza a in schoolchildren. Am. J. Clin. Nutr. 2010, 91, 1255-1260. [CrossRef]

5. Yakoob, M.Y.; Salam, R.A.; Khan, F.R.; Bhutta, Z.A. Vitamin D supplementation for preventing infections in children under five years of age. Cochrane Database Syst. Rev. 2016, 11, CD008824. [CrossRef]

6. Autier, P.; Mullie, P.; Macacu, A.; Dragomir, M.; Boniol, M.; Coppens, K.; Pizot, C.; Boniol, M. Effect of vitamin D supplementation on non-skeletal disorders: A systematic review of meta-analyses and randomised trials. Lancet Diabetes Endocrinol. 2017, 5, 986-1004. [CrossRef]

7. Holick, M.F.; Chen, T.C. Vitamin D deficiency: A worldwide problem with health consequences. Am. J. Clin. Nutr. 2008, 87, 1080S-1086S. [CrossRef] 
8. Von Hurst, P.R.; Stonehouse, W.; Coad, J. Vitamin D supplementation reduces insulin resistance in South Asian women living in New Zealand who are insulin resistant and vitamin D deficient-a randomised, placebo-controlled trial. Br. J. Nutr. 2010, 103, 549-555. [CrossRef]

9. Wang, T.J.; Pencina, M.J.; Booth, S.L.; Jacques, P.F.; Ingelsson, E.; Lanier, K.; Benjamin, E.J.; D'Agostino, R.B.; Wolf, M.; Vasan, R.S. Vitamin D deficiency and risk of cardiovascular disease. Circulation 2008, 117, $503-511$. [CrossRef]

10. Gandini, S.; Boniol, M.; Haukka, J.; Byrnes, G.; Cox, B.; Sneyd, M.J.; Mullie, P.; Autier, P. Meta-analysis of observational studies of serum 25-hydroxyvitamin D levels and colorectal, breast and prostate cancer and colorectal adenoma. Int. J. Cancer 2011, 128, 1414-1424. [CrossRef]

11. McDonnell, S.L.; Baggerly, C.; French, C.B.; Baggerly, L.L.; Garland, C.F.; Gorham, E.D.; Lappe, J.M.; Heaney, R.P. Serum 25-hydroxyvitamin D concentrations $\geq 40 \mathrm{ng} / \mathrm{mL}$ are associated with $>65 \%$ lower cancer risk: Pooled analysis of randomized trial and prospective cohort study. PLoS ONE 2016, 11, e0152441.

12. Buell, J.S.; Scott, T.M.; Dawson-Hughes, B.; Dallal, G.E.; Rosenberg, I.H.; Folstein, M.F.; Tucker, K.L. Vitamin $\mathrm{D}$ is associated with cognitive function in elders receiving home health services. J. Gerontol. Ser. A Biol. Sci. Med Sci. 2009, 64, 888-895. [CrossRef] [PubMed]

13. Knekt, P.; Kilkkinen, A.; Rissanen, H.; Marniemi, J.; Sääksjärvi, K.; Heliövaara, M. Serum vitamin D and the risk of Parkinson disease. Arch. Neurol. 2010, 67, 808-811. [CrossRef] [PubMed]

14. Wilkins, C.H.; Sheline, Y.I.; Roe, C.M.; Birge, S.J.; Morris, J.C. Vitamin D deficiency is associated with low mood and worse cognitive performance in older adults. Am. J. Geriatr. Psychiatry 2006, 14, 1032-1040. [CrossRef] [PubMed]

15. McGrath, J.J.; Burne, T.H.; Féron, F.; Mackay-Sim, A.; Eyles, D.W. Developmental vitamin D deficiency and risk of schizophrenia: A 10-year update. Schizophr. Bull. 2010, 36, 1073-1078. [CrossRef]

16. Perez-Ferre, N.; Torrejon, M.J.; Fuentes, M.; Fernandez, M.D.; Ramos, A.; Bordiu, E.; del Valle, L.; Rubio, M.A.; Bedia, A.R.; Montanez, C.; et al. Association of low serum 25-hydroxyvitamin D levels in pregnancy with glucose homeostasis and obstetric and newborn outcomes. Endocr. Pract. 2012, 18, 676-684. [CrossRef]

17. Wei, S.Q.; Qi, H.P.; Luo, Z.C.; Fraser, W.D. Maternal vitamin D status and adverse pregnancy outcomes: A systematic review and meta-analysis. J. Matern. Fetal Neonatal Med. 2013, 26, 889-899. [CrossRef]

18. Dabrowski, F.A.; Grzechocinska, B.; Wielgos, M. The role of vitamin D in reproductive health-A trojan horse or the golden fleece? Nutrients 2015, 7, 4139-4153. [CrossRef]

19. Mousa, A.; Abell, S.; Scragg, R.; de Courten, B. Vitamin D in reproductive health and pregnancy. Semin. Reprod. Med. 2016, 34, e1-e13. [CrossRef]

20. Marquina, C.; Mousa, A.; Scragg, R.; de Courten, B. Vitamin D and cardiometabolic disorders: A review of current evidence, genetic determinants and pathomechanisms. Obes. Rev. 2018, 20, 262-277. [CrossRef]

21. Guillot, X.; Semerano, L.; Saidenberg-Kermanac'h, N.; Falgarone, G.; Boissier, M.C. Vitamin D and inflammation. Jt. Bone Spine 2010, 77, 552-557. [CrossRef] [PubMed]

22. Alvarez, J.A.; Ashraf, A. Role of vitamin D in insulin secretion and insulin sensitivity for glucose homeostasis. Int. J. Endocrinol. 2010, 2010, 351385. [CrossRef] [PubMed]

23. Evans, K.N.; Bulmer, J.N.; Kilby, M.D.; Hewison, M. Vitamin D and placental-decidual function. J. Soc. Gynecol. Investig. 2004, 11, 263-271. [CrossRef] [PubMed]

24. Hahn, S.; Haselhorst, U.; Tan, S.; Quadbeck, B.; Schmidt, M.; Roesler, S.; Kimmig, R.; Mann, K.; Janssen, O.E. Low serum 25-hydroxyvitamin D concentrations are associated with insulin resistance and obesity in women with polycystic ovary syndrome. Exp. Clin. Endocrinol. Diabetes 2006, 114, 577-583. [CrossRef]

25. Clifton-Bligh, R.J.; McElduff, P.; McElduff, A. Maternal vitamin D deficiency, ethnicity and gestational diabetes. Diabet. Med. 2008, 25, 678-684. [CrossRef]

26. Lau, S.L.; Gunton, J.E.; Athayde, N.P.; Byth, K.; Cheung, N.W. Serum 25-hydroxyvitamin D and glycated haemoglobin levels in women with gestational diabetes mellitus. Med. J. Aust. 2011, 194, 334-337. [CrossRef]

27. Lepsch, J.; Eshriqui, I.; Farias, D.R.; Vaz, J.S.; Cunha Figueiredo, A.C.; Adegboye, A.R.A.; Brito, A.; Mokhtar, R.; Allen, L.H.; Holick, M.F.; et al. Association between early pregnancy vitamin D status and changes in serum lipid profiles throughout pregnancy. Metabolism 2017, 70, 85-97. [CrossRef]

28. Ringrose, J.S.; PausJenssen, A.M.; Wilson, M.; Blanco, L.; Ward, H.; Wilson, T.W. Vitamin D and hypertension in pregnancy. Clin. Investig. Med. 2011, 34, E147-E154. [CrossRef] 
29. Bobbitt, K.R.; Peters, R.M.; Li, J.; Rao, S.D.; Woodcroft, K.J.; Cassidy-Bushrow, A.E. Early pregnancy vitamin $\mathrm{D}$ and patterns of antenatal inflammation in African-American women. J. Reprod. Immunol. 2015, 107, 52-58. [CrossRef]

30. Xu, L.; Lee, M.; Jeyabalan, A.; Roberts, J.M. The relationship of hypovitaminosis D and IL-6 in preeclampsia. Am. J. Obs. Gynecol. 2014, 210, 149.e1-149.e7. [CrossRef]

31. Farrant, H.J.W.; Krishnaveni, G.V.; Hill, J.C.; Boucher, B.J.; Fisher, D.J.; Noonan, K.; Osmond, C.; Veena, S.R.; Fall, C.H.D. Vitamin D insufficiency is common in Indian mothers but is not associated with gestational diabetes or variation in newborn size. Eur. J. Clin. Nutr. 2009, 63, 646-652. [CrossRef] [PubMed]

32. Park, S.; Yoon, H.K.; Ryu, H.M.; Han, Y.J.; Lee, S.W.; Park, B.K.; Park, S.Y.; Yim, C.H.; Kim, S.H. Maternal vitamin D deficiency in early pregnancy is not associated with gestational diabetes mellitus development or pregnancy outcomes in Korean pregnant women in a prospective study. J. Nutr. Sci. Vitaminol. 2014, 60, 269-275. [CrossRef] [PubMed]

33. Zhang, M.X.; Pan, G.T.; Guo, J.F.; Li, B.Y.; Qin, L.Q.; Zhang, Z.L. Vitamin D deficiency increases the risk of gestational diabetes mellitus: A meta-analysis of observational studies. Nutrients 2015, 7, 8366-8375. [CrossRef] [PubMed]

34. Boyle, V.T.; Thorstensen, E.B.; Mourath, D.; Jones, M.B.; McCowan, L.M.E.; Kenny, L.C.; Baker, P.N. The relationship between 25-hydroxyvitamin D concentration in early pregnancy and pregnancy outcomes in a large, prospective cohort. Br. J. Nutr. 2016, 116, 1409-1415. [CrossRef] [PubMed]

35. Rodriguez, A.; Garcia-Esteban, R.; Basterretxea, M.; Lertxundi, A.; Rodriguez-Bernal, C.; Iniguez, C.; Rodriguez-Dehli, C.; Tardon, A.; Espada, M.; Sunyer, J.; et al. Associations of maternal circulating 25-hydroxyvitamin D3 concentration with pregnancy and birth outcomes. Bjog: Int. J. Obstet. Gynaecol. 2015, 122, 1695-1704. [CrossRef] [PubMed]

36. Schneuer, F.J.; Roberts, C.L.; Guilbert, C.; Simpson, J.M.; Algert, C.S.; Khambalia, A.Z.; Tasevski, V.; Ashton, A.W.; Morris, J.M.; Nassar, N. Effects of maternal serum 25-hydroxyvitamin D concentrations in the first trimester on subsequent pregnancy outcomes in an Australian population. Am. J. Clin. Nutr. 2014, 99, 287-295. [CrossRef] [PubMed]

37. Asemi, Z.; Karamali, M.; Esmaillzadeh, A. Favorable effects of vitamin D supplementation on pregnancy outcomes in gestational diabetes: A double blind randomized controlled clinical trial. Horm. Metab. Res. 2015, 47, 565-570. [CrossRef]

38. Asemi, Z.; Samimi, M.; Tabassi, Z.; Shakeri, H.; Esmaillzadeh, A. Vitamin D supplementation affects serum high-sensitivity c-reactive protein, insulin resistance, and biomarkers of oxidative stress in pregnant women. J. Nutr. 2013, 143, 1432-1438. [CrossRef]

39. Li, Q.; Xing, B. Vitamin D3-supplemented yogurt drink improves insulin resistance and lipid profiles in women with gestational diabetes mellitus: A randomized double blinded clinical trial. Ann. Nutr. Metab. 2016, 68, 285-290.

40. Mojibian, M.; Soheilykhah, S.; Fallah Zadeh, M.A.; Jannati Moghadam, M. The effects of vitamin D supplementation on maternal and neonatal outcome: A randomized clinical trial. Iran J. Reprod. Med. 2015, 13, 687-696.

41. Sablok, A.; Batra, A.; Thariani, K.; Batra, A.; Bharti, R.; Aggarwal, A.R.; Kabi, B.C.; Chellani, H. Supplementation of vitamin D in pregnancy and its correlation with feto-maternal outcome. Clin. Endocrinol. 2015, 83, 536-541. [CrossRef] [PubMed]

42. Yap, C.; Cheung, N.W.; Gunton, J.E.; Athayde, N.; Munns, C.F.; Duke, A.; McLean, M. Vitamin D supplementation and the effects on glucose metabolism during pregnancy: A randomized controlled trial. Diabetes Care 2014, 37, 1837-1844. [CrossRef] [PubMed]

43. Zerofsky, M.S.; Jacoby, B.N.; Pedersen, T.L.; Stephensen, C.B. Daily cholecalciferol supplementation during pregnancy alters markers of regulatory immunity, inflammation, and clinical outcomes in a randomized controlled trial. J. Nutr. 2016, 146, 2388-2397. [CrossRef] [PubMed]

44. Corcoy, R.; Mendoza, L.C.; Simmons, D.; Desoye, G.; Adelantado, J.M.; Chico, A.; Devlieger, R.; van Assche, A.; Galjaard, S.; Timmerman, D.; et al. The DALI vitamin D randomized controlled trial for gestational diabetes mellitus prevention: No major benefit shown besides vitamin D sufficiency. Clin. Nutr. 2020, 39, 976-984. [CrossRef] 
45. Jamilian, M.; Mirhosseini, N.; Eslahi, M.; Bahmani, F.; Shokrpour, M.; Chamani, M.; Asemi, Z. The effects of magnesium-zinc-calcium-vitamin D co-supplementation on biomarkers of inflammation, oxidative stress and pregnancy outcomes in gestational diabetes. BMC Pregnancy Childbirth 2019, 19, 107. [CrossRef]

46. Karamali, M.; Bahramimoghadam, S.; Sharifzadeh, F.; Asemi, Z. Magnesium-zinc-calcium-vitamin D co-supplementation improves glycemic control and markers of cardiometabolic risk in gestational diabetes: A randomized, double-blind, placebo-controlled trial. Appl. Physiol. Nutr. Metab. 2018, 43, 565-570. [CrossRef]

47. Mousa, A.; Misso, M.; Teede, H.; Scragg, R.; de Courten, B. Effect of vitamin D supplementation on inflammation: Protocol for a systematic review. BMJ Open 2016, 6, e010804. [CrossRef]

48. Nair, R.; Maseeh, A. Vitamin D: The "sunshine" vitamin. J. Pharm. Pharm. 2012, 3, 118-126.

49. Fernando, M.; Ellery, S.J.; Marquina, C.; Lim, S.; Naderpoor, N.; Mousa, A. Vitamin D-binding protein in pregnancy and reproductive health. Nutrients 2020, 12, 1489.

50. Zerwekh, J.E. Blood biomarkers of vitamin D status. Am. J. Clin. Nutr. 2008, 87, 1087S-1091S. [CrossRef]

51. Holick, M.F. Vitamin D status: Measurement, interpretation, and clinical application. Ann. Epidemiol. 2009, 19, 73-78. [CrossRef] [PubMed]

52. Ross, A.C.; Manson, J.E.; Abrams, S.A.; Aloia, J.F.; Brannon, P.M.; Clinton, S.K.; Durazo-Arvizu, R.A.; Gallagher, J.C.; Gallo, R.L.; Jones, G.; et al. The 2011 report on dietary reference intakes for calcium and vitamin D from the Institute of Medicine: What clinicians need to know. J. Clin. Endocrinol. Metab. 2011, 96, 53-58. [PubMed]

53. Brannon, P.M.; Picciano, M.F. Vitamin D in pregnancy and lactation in humans. Annu. Rev. Nutr. 2011, 31, 89-115. [CrossRef]

54. Van Schoor, N.M.; Lips, P. Worldwide vitamin D status. Best Pract. Res. Clin. Endocrinol. Metab. 2011, 25, 671-680. [PubMed]

55. Grant, W.B.; Karras, S.N.; Bischoff-Ferrari, H.A.; Annweiler, C.; Boucher, B.J.; Juzeniene, A.; Garland, C.F.; Holick, M.F. Do studies reporting ' $u$ '-shaped serum 25-hydroxyvitamin D-health outcome relationships reflect adverse effects? Derm. Endocrinol. 2016, 8, e1187349. [CrossRef]

56. Mousa, A.; Naderpoor, N.; Teede, H.J.; de Courten, M.P.; Scragg, R.; de Courten, B. Vitamin D and cardiometabolic risk factors and diseases. Minerva Endocrinol. 2015, 40, 213-230.

57. Ginde, A.A.; Liu, M.C.; Camargo, C.A., Jr. Demographic differences and trends of vitamin D insufficiency in the US population, 1988-2004. Arch. Intern. Med. 2009, 169, 626-632. [CrossRef]

58. Nowson, C.A.; McGrath, J.J.; Ebeling, P.R.; Haikerwal, A.; Daly, R.M.; Sanders, K.M.; Seibel, M.J.; Mason, R.S. Vitamin D and health in adults in Australia and New Zealand: A position statement. Med. J. Aust. 2012, 196, 686-687.

59. Luk, J.; Torrealday, S.; Neal Perry, G.; Pal, L. Relevance of vitamin D in reproduction. Hum. Reprod. 2012, 27, 3015-3027. [CrossRef]

60. Hollis, B.W.; Wagner, C.L. New insights into the vitamin D requirements during pregnancy. Bone Res. 2017, 5, 17030. [CrossRef]

61. Barrett, H.; McElduff, A. Vitamin D and pregnancy: An old problem revisited. Best Pract. Res. Clin. Endocrinol. Metab. 2010, 24, 527-539. [CrossRef] [PubMed]

62. Yu, C.K.H.; Sykes, L.; Sethi, M.; Teoh, T.G.; Robinson, S. Vitamin D deficiency and supplementation during pregnancy. Clin. Endocrinol. 2009, 70, 685-690. [CrossRef] [PubMed]

63. Holick, M.F.; Binkley, N.C.; Bischoff-Ferrari, H.A.; Gordon, C.M.; Hanley, D.A.; Heaney, R.P.; Murad, M.H.; Weaver, C.M. Evaluation, treatment, and prevention of vitamin D deficiency: An Endocrine Society clinical practice guideline. J. Clin. Endocrinol. Metab. 2011, 96, 1911-1930. [CrossRef] [PubMed]

64. Royal Australian and New Zealand College of Obstetricians and Gynaecologists (RANZCOG). Vitamin and Mineral Supplementation and Pregnancy; RANZCOG: Melbourne, Australia, 2015.

65. Zhang, C.; Qiu, C.; Hu, F.B.; David, R.M.; van Dam, R.M.; Bralley, A.; Williams, M.A. Maternal plasma 25-hydroxyvitamin D concentrations and the risk for gestational diabetes mellitus. PLoS ONE 2008, 3, e3753. [CrossRef]

66. Bodnar, L.M.; Catov, J.M.; Simhan, H.N.; Holick, M.F.; Powers, R.W.; Roberts, J.M. Maternal vitamin D deficiency increases the risk of preeclampsia. J. Clin. Endocrinol. Metab. 2007, 92, 3517-3522. 
67. Mousa, A.; Abell, S.K.; Shorakae, S.; Harrison, C.L.; Naderpoor, N.; Hiam, D.; Moreno-Asso, A.; Stepto, N.K.; Teede, H.J.; de Courten, B. Relationship between vitamin D and gestational diabetes in overweight or obese pregnant women may be mediated by adiponectin. Mol. Nutr. Food Res. 2017, 61, 1700488. [CrossRef]

68. American Diabetes Association. 2. Classification and diagnosis of diabetes: Standards of medical care in diabetes-2019. Diabetes Care 2019, 42, S13-S28.

69. Feig, D.S.; Berger, H.; Donovan, L.; Godbout, A.; Kader, T.; Keely, E.; Sanghera, R. Diabetes and pregnancy. Can. J. Diabetes 2018, 42 (Suppl S1), S255-S282. [CrossRef]

70. Metzger, B.; Gabbe, S.; Persson, B.; Buchanan, T.; Catalano, P.; Damm, P. International association of diabetes and pregnancy study groups consensus panel. International association of diabetes and pregnancy study groups recommendations on the diagnosis and classification of hyperglycemia in pregnancy. Diabetes Care 2010, 33, 676-682.

71. World Health Organization. Diagnostic Criteria and Classification of Hyperglycaemia First Detected in Pregnancy; WHO: Geneva, Switzerland, 2013.

72. Correa, A.; Bardenheier, B.; Elixhauser, A.; Geiss, L.S.; Gregg, E. Trends in prevalence of diabetes among delivery hospitalizations, United States, 1993-2009. Matern. Child Health J. 2015, 19, 635-642. [CrossRef]

73. Moses, R.G.; Morris, G.J.; Petocz, P.; San Gil, F.; Garg, D. The impact of potential new diagnostic criteria on the prevalence of gestational diabetes mellitus in Australia. Med. J. Aust. 2011, 194, 338-340. [CrossRef]

74. De Courten, B.; Mousa, A.; Naderpoor, N.; Teede, H.; de Courten, M.P.J.; Scragg, R. Vitamin D supplementation for the prevention of type 2 diabetes in overweight adults: Study protocol for a randomized controlled trial. Trials 2015, 16, 1-12. [CrossRef] [PubMed]

75. Mousa, A.; Naderpoor, N.; Teede, H.; Scragg, R.; de Courten, B. Vitamin D supplementation for improvement of chronic low-grade inflammation in patients with type 2 diabetes: A systematic review and meta-analysis of randomized controlled trials. Nutr. Rev. 2018, 76, 380-394. [CrossRef] [PubMed]

76. Zhang, Q.; Cheng, Y.; He, M.; Li, T.; Ma, Z.; Cheng, H. Effect of various doses of vitamin D supplementation on pregnant women with gestational diabetes mellitus: A randomized controlled trial. Exp. Ther. Med. 2016, 12, 1889-1895. [CrossRef] [PubMed]

77. Hossain, N.; Kanani, F.H.; Ramzan, S.; Kausar, R.; Ayaz, S.; Khanani, R.; Pal, L. Obstetric and neonatal outcomes of maternal vitamin D supplementation: Results of an open-label, randomized controlled trial of antenatal vitamin D supplementation in Pakistani women. J. Clin. Endocrinol. Metab. 2014, 99, 2448-2455. [CrossRef]

78. Asemi, Z.; Hashemi, T.; Karamali, M.; Samimi, M.; Esmaillzadeh, A. Effects of vitamin D supplementation on glucose metabolism, lipid concentrations, inflammation, and oxidative stress in gestational diabetes: A double-blind randomized controlled clinical trial. Am. J. Clin. Nutr. 2013, 98, 1425-1432. [CrossRef]

79. Soheilykhah, S.; Mojibian, M.; Moghadam, M.J.; Shojaoddiny-Ardekani, A. The effect of different doses of vitamin D supplementation on insulin resistance during pregnancy. Gynecol. Endocrinol. 2013, 29, 396-399. [CrossRef]

80. Tehrani, H.G.; Mostajeran, F.; Banihashemi, B. Effect of vitamin D supplementation on the incidence of gestational diabetes. Adv. Biomed. Res. 2017, 6, 79.

81. Karamali, M.; Beihaghi, E.; Mohammadi, A.A.; Asemi, Z. Effects of high-dose vitamin D supplementation on metabolic status and pregnancy outcomes in pregnant women at risk for pre-eclampsia. Horm. Metab. Res. 2015, 47, 867-872. [CrossRef]

82. Samimi, M.; Kashi, M.; Foroozanfard, F.; Karamali, M.; Bahmani, F.; Asemi, Z.; Hamidian, Y.; Talari, H.R.; Esmaillzadeh, A. The effects of vitamin D plus calcium supplementation on metabolic profiles, biomarkers of inflammation, oxidative stress and pregnancy outcomes in pregnant women at risk for pre-eclampsia. J. Hum. Nutr. Diet. 2016, 29, 505-515. [CrossRef]

83. Shahgheibi, S.; Farhadifar, F.; Pouya, B. The effect of vitamin D supplementation on gestational diabetes in high-risk women: Results from a randomized placebo-controlled trial. J. Res. Med Sci. 2016, $21,2$.

84. Asemi, Z.; Karamali, M.; Esmaillzadeh, A. Effects of calcium-vitamin D co-supplementation on glycaemic control, inflammation and oxidative stress in gestational diabetes: A randomised placebo-controlled trial. Diabetologia 2014, 57, 1798-1806. [CrossRef] [PubMed]

85. Yazdchi, R.; Gargari, B.P.; Asghari-Jafarabadi, M.; Sahhaf, F. Effects of vitamin D supplementation on metabolic indices and hs-CRP levels in gestational diabetes mellitus patients: A randomized, double-blinded, placebo-controlled clinical trial. Nutr. Res. Pract. 2016, 10, 328-335. [CrossRef] [PubMed] 
86. Scragg, R. Limitations of vitamin D supplementation trials: Why observational studies will continue to help determine the role of vitamin D in health. J. Steroid Biochem. Mol. Biol. 2018, 177, 6-9. [CrossRef] [PubMed]

87. Spedding, S.; Vanlint, S.; Morris, H.; Scragg, R. Does vitamin D sufficiency equate to a single serum 25-hydroxyvitamin D level or are different levels required for non-skeletal diseases? Nutrients 2013, 5, 5127-5139. [CrossRef] [PubMed]

88. Heaney, R.P.; Armas, L.A. Quantifying the vitamin D economy. Nutr. Rev. 2015, 73, 51-67. [CrossRef] [PubMed]

89. Heaney, R.P. Guidelines for optimizing design and analysis of clinical studies of nutrient effects. Nutr. Rev. 2014, 72, 48-54. [CrossRef] [PubMed]

90. Hollis, B.W.; Wagner, C.L. Clinical review: The role of the parent compound vitamin D with respect to metabolism and function: Why clinical dose intervals can affect clinical outcomes. J. Clin. Endocrinol. Metab. 2013, 98, 4619-4628.

91. Hollis, B.W.; Johnson, D.; Hulsey, T.C.; Ebeling, M.; Wagner, C.L. Vitamin D supplementation during pregnancy: Double-blind, randomized clinical trial of safety and effectiveness. J. Bone Miner. Res. 2011, 26, 2341-2357.

92. Wagner, C.L.; Hulsey, T.C.; Fanning, D.; Ebeling, M.; Hollis, B.W. High-dose vitamin D3 supplementation in a cohort of breastfeeding mothers and their infants: A 6-month follow-up pilot study. Breastfeed. Med. 2006, 1, 59-70.

93. Prentice, A.; Goldberg, G.R.; Schoenmakers, I. Vitamin D across the lifecycle: Physiology and biomarkers. Am. J. Clin. Nutr. 2008, 88, 500S-506S. [CrossRef]

94. Karras, S.N.; Anagnostis, P.; Naughton, D.; Annweiler, C.; Petroczi, A.; Goulis, D.G. Vitamin D during pregnancy: Why observational studies suggest deficiency and interventional studies show no improvement in clinical outcomes? A narrative review. J. Endocrinol. Investig. 2015, 38, 1265-1275. [CrossRef] [PubMed]

95. Uwitonze, A.M.; Razzaque, M.S. Role of magnesium in vitamin D activation and function. J. Am. Osteopath. Assoc. 2018, 118, 181-189. [CrossRef] [PubMed]

96. Vieth, R.; Chan, P.-C.R.; MacFarlane, G.D. Efficacy and safety of vitamin D3 intake exceeding the lowest observed adverse effect level. Am. J. Clin. Nutr. 2001, 73, 288-294. [CrossRef] [PubMed]

Publisher's Note: MDPI stays neutral with regard to jurisdictional claims in published maps and institutional affiliations.

(C) 2020 by the author. Licensee MDPI, Basel, Switzerland. This article is an open access article distributed under the terms and conditions of the Creative Commons Attribution (CC BY) license (http://creativecommons.org/licenses/by/4.0/). 